\title{
Wavefronts and global stability in a time-delayed population model with stage structure
}

\author{
By Stephen A. GouRley ${ }^{1}$ And YAng KuAnG \\ ${ }^{1}$ Department of Mathematics and Statistics, University of Surrey, \\ Guildford, Surrey GU2 7XH, UK (s.gourley@surrey.ac.uk) \\ ${ }^{2}$ Department of Mathematics, Arizona State University, \\ Tempe, AZ 85287, USA (kuang@asu.edu)
}

Received 30 July 2001; revised 12 August 2002; accepted 12 November 2002; published online 25 April 2003

We formulate and study a one-dimensional single-species diffusive-delay population model. The time delay is the time taken from birth to maturity. Without diffusion, the delay differential model extends the well-known logistic differential equation by allowing delayed constant birth processes and instantaneous quadratically regulated death processes. This delayed model is known to have simple global dynamics similar to that of the logistic equation. Through the use of a sub/supersolution pair method, we show that the diffusive delay model continues to generate simple global dynamics. This has the important biological implication that quadratically regulated death processes dramatically simplify the growth dynamics. We also consider the possibility of travelling wavefront solutions of the scalar equation for the mature population, connecting the zero solution of that equation with the positive steady state. Our main finding here is that our fronts appear to be all monotone, regardless of the size of the delay. This is in sharp contrast to the frequently reported findings that delay causes a loss of monotonicity, with the front developing a prominent hump in some other delay models.

Keywords: diffusive-delay differential equation; wavefront; characteristic equation; stage structure; population model

\section{Introduction}

It has become recognized in recent years that the well-known delayed logistic differential equation

$$
\frac{\mathrm{d} x(t)}{\mathrm{d} t}=r x(t)\left(1-\frac{x(t-\tau)}{K}\right),
$$

where $r, K$ and $\tau$ are positive parameters, and the more general distributed delay equation

$$
\frac{\mathrm{d} x(t)}{\mathrm{d} t}=r x(t)\left(1-\frac{1}{K} \int_{-\infty}^{t} k(t-s) x(s) \mathrm{d} s\right)
$$

have certain weaknesses as models for the evolution of a single species $x(t)$. Often, the use of such models is not so much that they have any real microscopic justification, but more a consequence of their having solution properties that mirror the ecosystem 


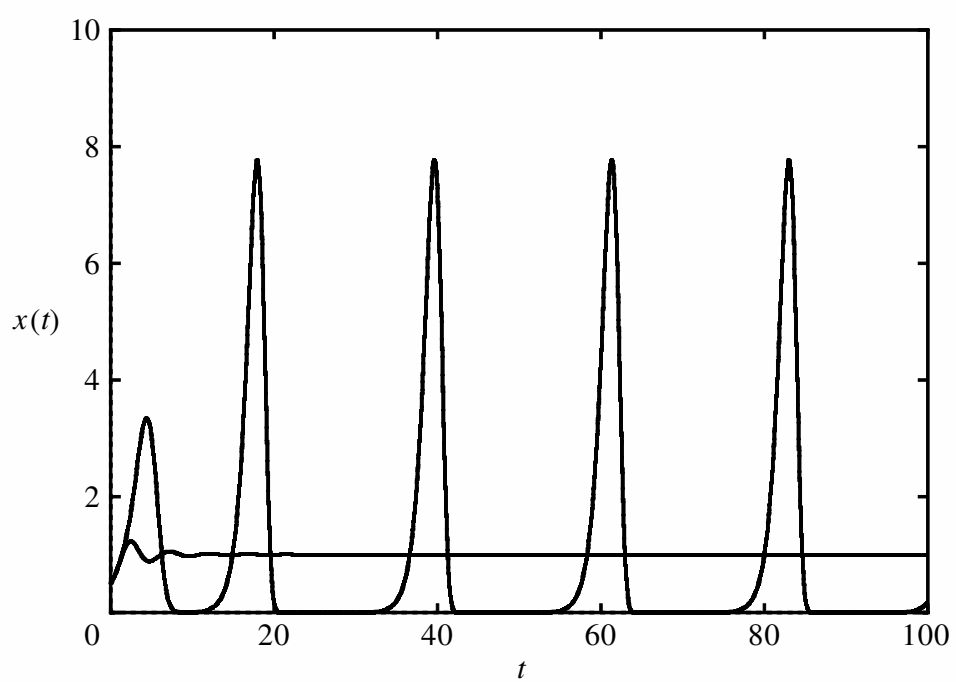

Figure 1. Solutions of the delayed logistic equation $x^{\prime}=x(1-x(t-\tau))$ for $\tau=1$ (stable) and $\tau=3$ (volatile).

under consideration. For example, to model an oscillating population, it is natural to consider the use of an equation that possesses periodic solutions. But although (1.1) does possess periodic solutions for $r \tau>\frac{1}{2} \pi$, it is well known that the amplitude of these oscillations increases extremely rapidly as $r \tau$ is increased, leading to an oscillation which when plotted shows an unrealistically large maximum value, and a minimum so low it is barely distinguishable from zero. This renders the assumption of the existence of a constant carrying capacity $K$ absurd and would even raise doubts about the continued validity of a continuum model at such times (see figure 1).

Also, it is frequently stated in the literature that because the equilibrium, or steady-state, solutions of the model equations will not depend on time, the time delays must be incorporated in such a way that the equilibrium solutions will not depend on them. However, the fact that the equilibrium solutions are not time dependent does not, in itself, mean they cannot depend on the delay parameters. As we illustrate below, in many ecological scenarios, it may be quite natural to have the equilibrium solutions showing some dependence on the delays. For example, consider the following system first introduced by Aiello \& Freedman (1990),

$$
\left.\begin{array}{rl}
\dot{u}_{\mathrm{i}}(t) & =\alpha u_{\mathrm{m}}(t)-\gamma u_{\mathrm{i}}(t)-\alpha \mathrm{e}^{-\gamma \tau} u_{\mathrm{m}}(t-\tau), \\
\dot{u}_{\mathrm{m}}(t) & =\alpha \mathrm{e}^{-\gamma \tau} u_{\mathrm{m}}(t-\tau)-\beta u_{\mathrm{m}}^{2}(t),
\end{array}\right\}
$$

where $\alpha, \beta, \gamma$ and the delay $\tau$ are positive constants. In this system, $u_{\mathrm{i}}$ and $u_{\mathrm{m}}$ denote, respectively, the numbers of immature and mature members of the population. The delay $\tau$ is the time taken from birth to maturity. The rate at which individuals are born is taken to be proportional to the number of matures at that time; this is the $\alpha u_{\mathrm{m}}(t)$ term. Death of immatures is modelled by the term $-\gamma u_{\mathrm{i}}(t)$. Death of matures is modelled by a quadratic term, as in the logistic equation. The term $\alpha \mathrm{e}^{-\gamma \tau} u_{\mathrm{m}}(t-\tau)$ appearing in both equations represents the rate at time $t$ at which individuals leave the immature and enter the mature class, having just reached maturity. These are individuals who were born at time $t-\tau$. Therefore, the rate of entering the mature 
class is $\alpha u_{\mathrm{m}}(t-\tau)$ times the fraction of those born at time $t-\tau$ who are still alive now. That this fraction is $\mathrm{e}^{-\gamma \tau}$ follows from the assumption that the death of immatures is following a linear law given by the term $-\gamma u_{\mathrm{i}}(t)$ (on the basis of such a law, if $N(t)$ is any population, then the number that survive from $t-\tau$ to $t$ is $\mathrm{e}^{-\gamma \tau} N(t-\tau)$ ). It is known that model (1.3) generates simple global dynamics (all positive solutions tend to the unique positive steady state) similar to that of the logistic equation (Aiello \& Freedman 1990). A subsequent paper of Aiello et al. (1992) extended this model to allow the time delay to be state dependent. However, their model proves to be too complicated for a thorough understanding.

System (1.3) possesses equilibrium states (steady states) given by

$$
\left(u_{\mathrm{i}}^{*}, u_{\mathrm{m}}^{*}\right)=(0,0) \text { and }\left(\frac{\alpha^{2}}{\beta \gamma} \mathrm{e}^{-\gamma \tau}\left(1-\mathrm{e}^{-\gamma \tau}\right), \frac{\alpha}{\beta} \mathrm{e}^{-\gamma \tau}\right),
$$

and it should be noted that the second of these depends on the delay $\tau$, as we should expect. After all, if the time from birth to maturity is long, then many of the immatures will not survive the juvenile phase, leading to a reduced mature adult population. Accordingly, it is not at all unnatural to have an equilibrium state showing a dependence on the delay.

It is our aim in this paper to study a diffusive version of the system (1.3) to allow for individuals moving around. When motion is allowed for, the derivation of the time-delayed term has to be altered to take proper account of the motion. Actually, the immatures may not move at all, especially if the immature phase is a larval phase, but our analysis allows for this possibility. If they do move, then certainly an individual is expected to enter the mature population class at a point in space different from where it was born. Taking account of this motion in re-deriving the time-delayed term is not necessarily a straightforward matter. It depends on the assumptions being made about the motion and also on the spatial domain. An infinite domain is slightly easier to handle than a finite one because, in the latter case, the individuals, as well as moving around, may have been interacting with the domain's boundaries. To keep the analysis as simple as possible, we shall assume the individuals are performing an unbiased random walk, so that the motion can be modelled in terms of Fickian diffusion as described in Murray (1989). Under this assumption, diffusion is approximated by adding Laplacian diffusion terms to the ordinary differential equations (ODEs) that model the temporal dynamics, and re-deriving the delay term as we describe below.

In the first instance, we shall work on the infinite one-dimensional spatial domain $x \in(-\infty, \infty)$. The case of a finite domain is slightly different and will be considered later. The immature and mature populations, $u_{\mathrm{i}}(t)$ and $u_{\mathrm{m}}(t)$, are now population densities $u_{\mathrm{i}}(x, t)$ and $u_{\mathrm{m}}(x, t)$, respectively. We argue that the delayed term $\alpha \mathrm{e}^{-\gamma \tau} u_{\mathrm{m}}(t-\tau)$ appearing twice in system (1.3), and representing the rate of leaving the immature and entering the mature class, must be replaced by

$$
\int_{-\infty}^{\infty} \frac{1}{\sqrt{4 \pi d_{\mathrm{i}} \tau}} \mathrm{e}^{-(x-y)^{2} / 4 d_{\mathrm{i}} \tau} \mathrm{e}^{-\gamma \tau} \alpha u_{\mathrm{m}}(y, t-\tau) \mathrm{d} y,
$$

where $d_{\mathrm{i}}>0$ is the diffusivity of the immature species. If the immatures are not, in fact, moving, then we would allow $d_{\mathrm{i}} \rightarrow 0$ in (1.5) to conclude that the corresponding expression is $\mathrm{e}^{-\gamma \tau} \alpha u_{\mathrm{m}}(x, t-\tau)$. If $d_{\mathrm{i}}>0$, expression (1.5) allows for the fact that an 
individual that enters the mature class at position $x$ will most likely have been born at some other point $y$. So, according to the assumptions about births and deaths made in our earlier discussion about system (1.3), the total rate of entering the mature class at time $t$ and position $x$ is therefore now made up as follows,

$$
\begin{aligned}
& \alpha u_{\mathrm{m}}(y, t-\tau)=\text { number born at } y, \\
& \mathrm{e}^{-\gamma \tau} \alpha u_{\mathrm{m}}(y, t-\tau)=\text { number born at } y \text { and still alive now, } \\
& \frac{1}{\sqrt{4 \pi d_{\mathrm{i}} \tau}} \mathrm{e}^{-(x-y)^{2} / 4 d_{\mathrm{i}} \tau} \mathrm{e}^{-\gamma \tau} \alpha u_{\mathrm{m}}(y, t-\tau)=\text { number born at } y \text { still alive } \\
& \text { and now at } x
\end{aligned}
$$

and finally the integral totals up the contributions from all parts of the domain. Note that of those individuals born at $y$ and still alive, to calculate the number that are now at $x$, we are multiplying by $g(x, y, t=\tau)$, where $g(x, y, t)$ satisfies the linear diffusion equation $\partial g / \partial t=d_{\mathrm{i}} \partial^{2} g / \partial x^{2},-\infty<x<\infty$, with the initial condition $g(x, y, 0)=\delta(x-y)$. These arguments reflect the assumptions we have made about the diffusivity being linear (Fickian) diffusion. See Gourley \& Britton (1996) for more detailed discussions of these points. The reader will also realize that, on a finite domain, the use of this heat kernel cannot allow for interactions with the domain's boundaries and will therefore be inappropriate. Instead, a more complicated kernel must be used. This point will be discussed later.

Thus we initially study the model

$$
\left.\begin{array}{rl}
\frac{\partial u_{\mathrm{i}}}{\partial t} & =d_{\mathrm{i}} \frac{\partial^{2} u_{\mathrm{i}}}{\partial x^{2}}+\alpha u_{\mathrm{m}}-\gamma u_{\mathrm{i}}-\alpha \mathrm{e}^{-\gamma \tau} \int_{-\infty}^{\infty} \frac{1}{\sqrt{4 \pi d_{\mathrm{i}} \tau}} \mathrm{e}^{-(x-y)^{2} / 4 d_{\mathrm{i}} \tau} u_{\mathrm{m}}(y, t-\tau) \mathrm{d} y, \\
\frac{\partial u_{\mathrm{m}}}{\partial t} & =d_{\mathrm{m}} \frac{\partial^{2} u_{\mathrm{m}}}{\partial x^{2}}+\alpha \mathrm{e}^{-\gamma \tau} \int_{-\infty}^{\infty} \frac{1}{\sqrt{4 \pi d_{\mathrm{i}} \tau}} \mathrm{e}^{-(x-y)^{2} / 4 d_{\mathrm{i}} \tau} u_{\mathrm{m}}(y, t-\tau) \mathrm{d} y-\beta u_{\mathrm{m}}^{2}
\end{array}\right\}
$$

for $x \in(-\infty, \infty)$ and $t>0$. While our analysis makes no particular assumptions as to the relative magnitudes of the diffusivities $d_{\mathrm{i}}, d_{\mathrm{m}}$, two possibilities of particular interest ecologically are (i) $d_{\mathrm{i}}=0$, meaning the immatures do not move, and (ii) $d_{\mathrm{i}}=d_{\mathrm{m}}$, which will be the case when the immatures stay with their parents. It should be noted that (1.6) is not a fully coupled system in that the second equation, for the mature population $u_{\mathrm{m}}$, can be solved independently of the first. Consideration of this second equation alone is an interesting and non-trivial mathematical problem in its own right.

The main purpose of this paper is to examine how the solutions of the diffusive system (1.6) (and, in particular, the solution of the second equation thereof, considered independently of the first) compare with the solutions of other delayed diffusion models of population dynamics, such as the diffusive Hutchinson's equation

$$
\frac{\partial u}{\partial t}(x, t)=\frac{\partial^{2} u}{\partial x^{2}}(x, t)+r u(x, t)\left(1-\frac{u(x, t-\tau)}{K}\right) .
$$

Starting with the infinite domain system (1.6), we shall analyse the linear stability of the system about its two spatially uniform equilibrium solutions (the equilibria of (1.6) are the same as those of (1.3) and are given by (1.4)). Then we consider the possibility of travelling wavefront solutions of the scalar equation for the mature population $u_{\mathrm{m}}$ connecting the zero solution of that equation with the 
$u_{\mathrm{m}}=(\alpha / \beta) \exp (-\gamma \tau)$ state. These fronts will be compared with the results of some recent studies of travelling-front solutions of other scalar delay equations with diffusion such as (1.7). Our main finding here is that our fronts are apparently all monotone, regardless of the size of the delay $\tau$ (investigators of other delay problems frequently have reported that increasing the delay causes a loss of monotonicity, with the front developing a prominent hump).

Then the case of a finite domain will be discussed. The time-delay term must be re-derived using a different spatial distribution kernel. Our main result here is a powerful global convergence theorem, which holds without any restriction on the parameters other than that they be positive.

\section{Linear stability and wavefronts}

We start by linearizing (1.6) about the non-zero equilibrium state. The zero state will be discussed later. Using methods now accepted as standard, we find, after some algebra, that the linearized equations admit non-trivial solutions of the form $\left(c_{1}, c_{2}\right) \exp (\sigma t+\mathrm{i} k x)$ if and only if the determinant

$$
\left|\begin{array}{cc}
\sigma+\gamma+d_{\mathrm{i}} k^{2} & -\alpha+\alpha \mathrm{e}^{-\gamma \tau} \mathrm{e}^{-\sigma \tau} \mathrm{e}^{-d_{\mathrm{i}} k^{2} \tau} \\
0 & \sigma-\alpha \mathrm{e}^{-\gamma \tau} \mathrm{e}^{-\sigma \tau} \mathrm{e}^{-d_{\mathrm{i}} k^{2} \tau}+2 \alpha \mathrm{e}^{-\gamma \tau}+d_{\mathrm{m}} k^{2}
\end{array}\right|=0 .
$$

The roots $\sigma$ of this dispersion relation are $-\gamma-d_{\mathrm{i}} k^{2}(<0)$, together with the roots of the transcendental equation

$$
\sigma-\alpha \mathrm{e}^{-\gamma \tau} \mathrm{e}^{-\sigma \tau} \mathrm{e}^{-d_{\mathrm{i}} k^{2} \tau}+2 \alpha \mathrm{e}^{-\gamma \tau}+d_{\mathrm{m}} k^{2}=0 .
$$

Note that this analysis is simplified by the system not being fully coupled. The dispersion relation (2.1) is, in fact, what one would obtain from the equation for the mature population $u_{\mathrm{m}}$ only, treated independently of the $u_{\mathrm{i}}$ equation. Even so, note that both diffusivities $d_{\mathrm{i}}$ and $d_{\mathrm{m}}$ are still present. As far as stability is concerned, if it is true that, for every wavenumber $k \geqslant 0$, the roots of (2.1) all lie in the left half of the complex plane, i.e. satisfy $\operatorname{Re} \sigma<0$, then we may declare that the non-zero steady state of (1.6) is linearly stable. We now show that this is, in fact, the case for any positive values of the other parameters. Certainly, when the delay $\tau$ is zero, equation (2.1) has only one real negative root $-\alpha-d_{\mathrm{m}} k^{2}$. Standard theory tells us that if we increase $\tau$ from zero new roots will appear, which, for small $\tau$, will be in the left half of the complex plane. We must investigate whether any of these roots cross the imaginary axis as $\tau$ is further increased. To investigate this possibility, set $\sigma=\mathrm{i} \omega$ with $\omega$ real and positive. Substituting into (2.1), taking the complex conjugate and then eliminating $\exp (\mathrm{i} \omega \tau)$ terms yields

$$
\omega^{2}+4 \alpha^{2} \mathrm{e}^{-2 \gamma \tau}+4 \alpha d_{\mathrm{m}} k^{2} \mathrm{e}^{-\gamma \tau}+d_{\mathrm{m}}^{2} k^{4}=\alpha^{2} \mathrm{e}^{-2 \gamma \tau} \mathrm{e}^{-2 d_{\mathrm{i}} k^{2} \tau} \leqslant \alpha^{2} \mathrm{e}^{-2 \gamma \tau},
$$

giving $\omega^{2}+3 \alpha^{2} \mathrm{e}^{-2 \gamma \tau}+4 \alpha d_{\mathrm{m}} k^{2} \mathrm{e}^{-\gamma \tau}+d_{\mathrm{m}}^{2} k^{4} \leqslant 0$, which is impossible. Our conclusion, therefore, is that the non-zero steady state is linearly stable, independently of the size of the delay and other parameters and, in particular, it is not destabilized by diffusion. For the corresponding ODE system (1.3) (which arises as a special case of (1.6) when we restrict to the sub-class of spatially uniform solutions), it is known (see Aiello \& Freedman 1990; Kuang 1993) that the non-zero steady state is, in fact, globally asymptotically stable for all values of the parameters. 
By using similar ideas, it is straightforward to show that the zero steady state $\left(u_{\mathrm{i}}^{*}, u_{\mathrm{m}}^{*}\right)=(0,0)$ of $(1.6)$ is always unstable.

Concentrating now on the second equation of (1.6) only, and motivated by the results of the linearized analysis we have just carried out, it is natural to inquire into the possibility of travelling wave-front solutions connecting the zero solution to the $u_{\mathrm{m}}=(\alpha / \beta) \exp (-\gamma \tau)$ solution of that equation. We must stress from the outset that we do not have conclusive proof of the existence of such a solution. So et al. (2001) proved existence of a travelling-front solution to another equation with spatio-temporal delay, but their proof does depend heavily on the structure of their particular equation and their ideas do not seem to adapt to the equation of the present paper. However, some asymptotic analysis of the full nonlinear problem and examination of the linearized equations as $x \rightarrow \pm \infty$ suggest the existence of a front that is monotone for all values of the parameters, including the delay $\tau$. This is in contrast to analytical and numerical studies of other delay and non-local reactiondiffusion equations, which can have non-monotone fronts for sufficiently large delays (see, for example, Ashwin et al. 2002; Gourley 2000a, b; So et al. 2001).

To seek a travelling-front solution of the second equation of $(1.6)$, we set $u_{\mathrm{m}}(x, t)=$ $U(z)$, where $z=x+c t$ and $c \geqslant 0$ without loss of generality. The ODE to be satisfied by $U(z)$ is

$$
c U^{\prime}(z)=\alpha \mathrm{e}^{-\gamma \tau} \int_{-\infty}^{\infty} \frac{1}{\sqrt{4 \pi d_{\mathrm{i}} \tau}} \mathrm{e}^{-y^{2} / 4 d_{\mathrm{i}} \tau} U(z-c \tau-y) \mathrm{d} y-\beta U^{2}(z)+d_{\mathrm{m}} U^{\prime \prime}(z)
$$

with

$$
U(-\infty)=0 \quad \text { and } \quad U(\infty)=\frac{\alpha}{\beta} \mathrm{e}^{-\gamma \tau} .
$$

A solution of (2.2), (2.3) corresponds to a leftward-moving travelling-front solution moving with speed $c$. Motivated by ecological considerations, we are interested only in solutions that are non-negative for all $z$. We can obtain a necessary condition on the front speed $c$ by linearizing (2.2) ahead of the front, i.e. for $z \rightarrow-\infty$. The linearized equation will be

$$
c U^{\prime}(z)=\alpha \mathrm{e}^{-\gamma \tau} \int_{-\infty}^{\infty} \frac{1}{\sqrt{4 \pi d_{\mathrm{i}} \tau}} \mathrm{e}^{-y^{2} / 4 d_{\mathrm{i}} \tau} U(z-c \tau-y) \mathrm{d} y+d_{\mathrm{m}} U^{\prime \prime}(z) .
$$

Seeking solutions of this proportional to $\exp (\lambda z)$, we find that $\lambda$ satisfies

$$
c \lambda-d_{\mathrm{m}} \lambda^{2}=\alpha \mathrm{e}^{-\gamma \tau} \mathrm{e}^{-\lambda c \tau} \mathrm{e}^{\lambda^{2} d_{\mathrm{i}} \tau}
$$

If $\tau$ is zero, equation (2.4) reduces to a quadratic equation and the nonlinear equation (2.2) reduces to $c U^{\prime}=\alpha U-\beta U^{2}+d_{\mathrm{m}} U^{\prime \prime}$. Using standard phase-plane arguments, we find that the minimum wave speed $c$ for having a solution that is non-negative for all $z$ is $2 \sqrt{\alpha d_{\mathrm{m}}}$. Now assume that $\tau$ is positive. In order to have a front $U(z)$ that tends to 0 as $z \rightarrow-\infty$ without oscillating, it will be necessary for (2.4) to have some real positive roots. The onset of oscillations is associated with a total loss of all real positive roots of (2.4). Figure 2 shows a plot of the left- and right-hand sides of (2.4) as functions of $\lambda$, showing a situation giving two real positive roots.

Generically, there are either two real positive roots or none, and it is clear that the latter situation can be brought about by changing the values of certain parameters. 


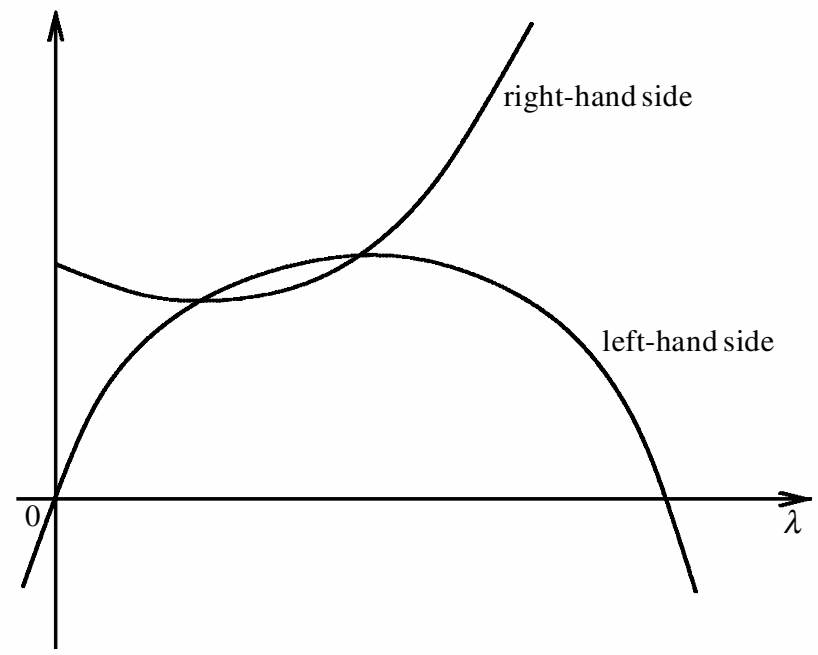

Figure 2. Graphs of the left- and right-hand sides of (2.4) as functions of $\lambda$.

The critical situation (determining the minimum wave speed $c$ ) is when the two curves touch, so that there is just one repeated root, and this happens when

$$
\left.\begin{array}{rl}
c \lambda_{*}-d_{\mathrm{m}} \lambda_{*}^{2} & =\alpha \mathrm{e}^{-\gamma \tau} \mathrm{e}^{-\lambda_{*} c \tau} \mathrm{e}^{\lambda_{*}^{2} d_{\mathrm{i}} \tau}, \\
c-2 d_{\mathrm{m}} \lambda_{*} & =\alpha \mathrm{e}^{-\gamma \tau}\left(2 \lambda_{*} d_{\mathrm{i}} \tau-c \tau\right) \mathrm{e}^{-\lambda_{*} c \tau} \mathrm{e}^{\lambda_{*}^{2} d_{\mathrm{i}} \tau},
\end{array}\right\}
$$

where $\lambda_{*}$ is the single repeated root. On elimination of the exponential terms, we find that $\lambda_{*}$ must satisfy the cubic equation

$$
f\left(\lambda_{*}\right):=2 d_{\mathrm{i}} d_{\mathrm{m}} \tau \lambda_{*}^{3}-\left(c \tau d_{\mathrm{m}}+2 c \tau d_{\mathrm{i}}\right) \lambda_{*}^{2}+\left(c^{2} \tau-2 d_{\mathrm{m}}\right) \lambda_{*}+c=0 .
$$

It is straightforward to see that this cubic equation always has one real negative root and two real positive roots, one less than and one greater than $c / d_{\mathrm{m}}$ (that the latter is so follows from the fact that $\left.f\left(c / d_{\mathrm{m}}\right)=-c<0\right)$. The root that is larger than $c / d_{\mathrm{m}}$ cannot satisfy the first equation of (2.5). Since $\lambda_{*}>0$, we conclude that $\lambda_{*}$ must be the smaller of the two positive roots of (2.6). Knowing $\lambda_{*}$, the value of $c$ given implicitly by either equation of $(2.5)$, which we now call $c_{\min }(\tau)$, is the minimum wave speed. Unfortunately, the minimum speed cannot be computed explicitly.

We do know that when $\tau=0$ the minimum speed is $2 \sqrt{\alpha d_{\mathrm{m}}}$. It is of interest to inquire whether the minimum speed will decrease or increase when delay is introduced, and we can gain useful information on this using a perturbation analysis for small $\tau$. Note that $\lambda_{*}$ depends on $\tau$ too. Therefore, we write

$$
\begin{aligned}
c_{\min }(\tau) & =c_{\min }^{(0)}+\tau c_{\min }^{(1)}+\tau^{2} c_{\min }^{(2)}+\cdots, \\
\lambda_{*} & =\lambda_{*}^{(0)}+\tau \lambda_{*}^{(1)}+\tau^{2} \lambda_{*}^{(2)}+\cdots,
\end{aligned}
$$

where $c_{\min }^{(0)}=2 \sqrt{\alpha d_{\mathrm{m}}}$ and $\lambda_{*}^{(0)}=c_{\min }^{(0)} / 2 d_{\mathrm{m}}$. After some algebra, we find that

$$
c_{\min }^{(1)}=\sqrt{\frac{\alpha}{d_{\mathrm{m}}}}\left(\alpha d_{\mathrm{i}}-2 \alpha d_{\mathrm{m}}-\gamma d_{\mathrm{m}}\right),
$$

so that, for small delays, the minimum speed is given by

$$
c_{\min }(\tau)=2 \sqrt{\alpha d_{\mathrm{m}}}+\tau \sqrt{\frac{\alpha}{d_{\mathrm{m}}}}\left(\alpha d_{\mathrm{i}}-2 \alpha d_{\mathrm{m}}-\gamma d_{\mathrm{m}}\right)+\cdots .
$$


Thus, whether the speed is increased or reduced by a small delay $\tau$ depends on the sign of $\alpha d_{\mathrm{i}}-2 \alpha d_{\mathrm{m}}-\gamma d_{\mathrm{m}}$. As we mentioned before, we consider the cases $d_{\mathrm{i}}=0$ and $d_{\mathrm{i}}=d_{\mathrm{m}}$ to be of particular relevance ecologically. In either of these cases, $c_{\min }^{(1)}<0$, so that the wave is slowed down by the delay. More generally, it will be slowed down if $\alpha d_{\mathrm{i}}<2 \alpha d_{\mathrm{m}}+\gamma d_{\mathrm{m}}$.

Motivated by studies of fronts in delay equations by other investigators, we are interested in whether the travelling-front solutions are monotone or not. We shall now present a strong case (though not a conclusive proof) that our fronts are monotone for all values of the delay $\tau \geqslant 0$. We shall do this (i) by studying the linearization at the other end of the front (the rear), and (ii) by computing a uniformly valid asymptotic expression for the front when the speed $c \rightarrow \infty$ and proving that the first term of this is indeed monotone for all $z$.

To investigate the linearization at the rear of the front, where $z \rightarrow \infty$ and $U(z) \rightarrow$ $(\alpha / \beta) \exp (-\gamma \tau)$, we set

$$
U(z)=\frac{\alpha}{\beta} \exp (-\gamma \tau)+V(z)
$$

substitute into (2.2) and linearize to obtain

$$
c V^{\prime}(z)=\alpha \mathrm{e}^{-\gamma \tau} \int_{-\infty}^{\infty} \frac{1}{\sqrt{4 \pi d_{\mathrm{i}} \tau}} \mathrm{e}^{-y^{2} / 4 d_{\mathrm{i}} \tau} V(z-c \tau-y) \mathrm{d} y-2 \alpha \mathrm{e}^{-\gamma \tau} V(z)+d_{\mathrm{m}} V^{\prime \prime}(z) .
$$

Solutions proportional to $\exp (\lambda z)$ exist whenever $\lambda$ satisfies

$$
c \lambda-d_{\mathrm{m}} \lambda^{2}=\alpha \mathrm{e}^{-\gamma \tau} \mathrm{e}^{-\lambda c \tau} \mathrm{e}^{\lambda^{2} d_{\mathrm{i}} \tau}-2 \alpha \mathrm{e}^{-\gamma \tau} .
$$

Since all this is for $z \rightarrow \infty$, monotonicity now requires the above equation to have real negative roots. Oscillations would set in if all such roots were lost, and this is the situation in which other investigators have observed 'humps' and oscillations in the travelling-front solutions of their delay models. However, the reader can easily verify, by plotting the left- and right-hand sides of (2.8) against $\lambda$, that, in fact, equation (2.8) always has one real negative root for any positive values of the parameters. This suggests that oscillations never set in and that the front probably approaches $(\alpha / \beta) \exp (-\gamma \tau)$ monotonically as $z \rightarrow \infty$ for any delay $\tau \geqslant 0$.

To gain further evidence for the above-mentioned remarks on the front's likely behaviour, we now aim to construct a uniformly valid asymptotic approximation to our front for large values of the speed $c$. Our approach here is based on ideas of Canosa (1973), who obtained a similar such approximation to the travelling-front solution of the well-known Fisher equation. Although Canosa's analysis was carried out for $c \rightarrow \infty$, it is known that, even if the speed $c$ is given its lowest ecologically relevant value (i.e. the minimum speed for positive solutions), the first term alone of the asymptotic solution for Fisher's equation is within a few percent of the true solution (Murray 1989). We shall use the approach here to gain further evidence for the monotonicity of our fronts. This asymptotic approach for large wavespeeds has recently been shown to also be of value in studying certain coupled systems (Sherratt 2000).

Following the approach of Canosa (1973), we shall assume $c$ is large, introduce the small parameter

$$
\varepsilon=\frac{1}{c^{2}}
$$


and seek a solution of $(2.2)$ of the form $U(z)=\tilde{U}(\tilde{z})$, where $\tilde{z}=\sqrt{\varepsilon} z=z / c$. Under this transformation, equation $(2.2)$ becomes

$$
\tilde{U}^{\prime}(\tilde{z})=\alpha \mathrm{e}^{-\gamma \tau} \int_{-\infty}^{\infty} \frac{1}{\sqrt{4 \pi d_{\mathrm{i}} \tau}} \mathrm{e}^{-y^{2} / 4 d_{\mathrm{i}} \tau} \tilde{U}(\tilde{z}-\tau-\sqrt{\varepsilon} y) \mathrm{d} y-\beta \tilde{U}^{2}(\tilde{z})+\varepsilon d_{\mathrm{m}} \tilde{U}^{\prime \prime}(\tilde{z}) .
$$

This can be approximated by Taylor expanding $\tilde{U}(\tilde{z}-\tau-\sqrt{\varepsilon} y)$ about $\tilde{z}-\tau$ to yield the following delay differential equation,

$$
\tilde{U}^{\prime}(\tilde{z})=\alpha \mathrm{e}^{-\gamma \tau}\left[\tilde{U}(\tilde{z}-\tau)+\varepsilon d_{\mathrm{i}} \tau \tilde{U}^{\prime \prime}(\tilde{z}-\tau)+O\left(\varepsilon^{2}\right)\right]-\beta \tilde{U}^{2}(\tilde{z})+\varepsilon d_{\mathrm{m}} \tilde{U}^{\prime \prime}(\tilde{z}),
$$

which has to be solved subject to

$$
\tilde{U}(-\infty)=0 \quad \text { and } \quad \tilde{U}(\infty)=\frac{\alpha}{\beta} \mathrm{e}^{-\gamma \tau} .
$$

We seek a solution of the form

$$
\tilde{U}(\tilde{z})=\tilde{U}_{0}(\tilde{z})+\varepsilon \tilde{U}_{1}(\tilde{z})+\cdots .
$$

Substituting into (2.10) and comparing powers of $\varepsilon^{0}$ yields the finding that $\tilde{U}_{0}$ must satisfy

$$
\tilde{U}_{0}^{\prime}(\tilde{z})=\alpha \mathrm{e}^{-\gamma \tau} \tilde{U}_{0}(\tilde{z}-\tau)-\beta \tilde{U}_{0}^{2}(\tilde{z}),
$$

with

$$
\tilde{U}_{0}(-\infty)=0 \quad \text { and } \quad \tilde{U}_{0}(\infty)=\frac{\alpha}{\beta} \mathrm{e}^{-\gamma \tau} .
$$

Problem (2.12), (2.13) is invariant to translations in $\tilde{z}$. The solution is unique up to these translations. The slight non-uniqueness is not a problem for us, since we are concerned primarily with monotonicity, but we can, if we wish, eliminate the non-uniqueness by requiring that

$$
\tilde{U}_{0}(0)=\frac{\alpha}{2 \beta} \exp (-\gamma \tau)
$$

The solution $\tilde{U}_{0}(\tilde{z})$ is the lowest-order term in the asymptotic expression $(2.11)$ and what we shall now do is prove the following theorem concerning monotonicity of $\tilde{U}_{0}(\tilde{z})$ as $z \rightarrow-\infty$. It is already known (Aiello \& Freedman 1990) that all positive solutions of $(2.12)$ tend to $(\alpha / \beta) \mathrm{e}^{-\gamma \tau}$ as $\tilde{z} \rightarrow \infty$.

Theorem 2.1. Any positive solution $\tilde{U}_{0}(\tilde{z})$ of (2.12) subject to (2.13) is a monotonic non-decreasing function of $\tilde{z}$ for all $-\infty<\tilde{z}<\infty$.

Proof. For notational convenience, we restate and renumber (2.12), (2.13) without the tildes and subscript on $U$,

$$
U^{\prime}(z)=\alpha \mathrm{e}^{-\gamma \tau} U(z-\tau)-\beta U^{2}(z), \quad U(-\infty)=0, \quad U(\infty)=\frac{\alpha}{\beta} \mathrm{e}^{-\gamma \tau} .
$$

The proof of the theorem is in three stages. We prove that

(i) the decay of a positive solution of (2.14) to zero as $z \rightarrow-\infty$ is strictly monotone;

(ii) a positive solution takes values in the range $[0,(\alpha / \beta) \exp (-\gamma \tau)]$ only; and, finally,

(iii) a positive solution is monotone non-decreasing. 
To prove (i), we let

$$
\delta(\theta)=\beta^{-1} \alpha \exp \{-\gamma \tau-\alpha \tau \exp \{(\beta \theta-\gamma) \tau\}\}
$$

and choose $\varepsilon \in(0, \min \{1, \delta(1)\})$. There exists a $z_{\varepsilon}$ such that $z \leqslant z_{\varepsilon}$ implies that $0<U(z)<\varepsilon$. Hence, for $z \leqslant z_{\varepsilon}$, we have

$$
U^{\prime}(z) \geqslant-\beta U^{2}(z) \geqslant-\beta \varepsilon U(z),
$$

which yields $U(z) \geqslant U(z-\tau) \mathrm{e}^{-\beta \varepsilon \tau}$ or, equivalently,

$$
U(z-\tau) \leqslant U(z) \mathrm{e}^{\beta \varepsilon \tau} .
$$

Substituting this into the equation of (2.14) leads to

$$
U^{\prime}(z) \leqslant \alpha \mathrm{e}^{(\beta \varepsilon-\gamma) \tau} U(z)-\beta U^{2}(z) \leqslant \alpha \mathrm{e}^{(\beta \varepsilon-\gamma) \tau} U(z),
$$

which yields

$$
U(z) \leqslant U(z-\tau) \mathrm{e}^{\alpha \tau \exp \{(\beta \varepsilon-\gamma) \tau\}} .
$$

Hence we have, for $z \leqslant z_{\varepsilon}$,

$$
U(z-\tau) \geqslant U(z) \mathrm{e}^{-\alpha \tau \exp \{(\beta \varepsilon-\gamma) \tau\}}
$$

and

$$
U^{\prime}(z) \geqslant \alpha \mathrm{e}^{-\gamma \tau-\alpha \tau \exp \{(\beta \varepsilon-\gamma) \tau\}} U(z)-\beta U^{2}(z) .
$$

Therefore, $U^{\prime}(z)>0$ if $z \leqslant z_{\varepsilon}$ and

$$
U(z)<\beta^{-1} \alpha \exp \{-\gamma \tau-\alpha \tau \exp \{(\beta \varepsilon-\gamma) \tau\}\}=\delta(\varepsilon) .
$$

We now show that $U(z)<\delta(\varepsilon)$ follows automatically from $z \leqslant z_{\varepsilon}$. Notice that $\delta(\varepsilon)$ is strictly decreasing. Hence, since $\varepsilon<1$, we have $\delta(\varepsilon)>\delta(1)$. Therefore, if $z \leqslant z_{\varepsilon}$, we have $U(z)<\varepsilon<\min \{1, \delta(1)\}<\delta(\varepsilon)$, as desired. Thus $z \leqslant z_{\varepsilon}$ implies $U^{\prime}(z)>0$, proving (i).

To prove (ii), suppose that there exists a point where $U>(\alpha / \beta) \exp (-\gamma \tau)$. Then $U$ must attain a global maximum $z_{2}$. At $z_{2}$, we have the fact that $U^{\prime}\left(z_{2}\right)=0$ and $U\left(z_{2}\right)>(\alpha / \beta) \exp (-\gamma \tau)$. Then

$$
\begin{aligned}
0=U^{\prime}\left(z_{2}\right) & =\alpha \mathrm{e}^{-\gamma \tau} U\left(z_{2}-\tau\right)-\beta U^{2}\left(z_{2}\right) \\
& \leqslant \alpha \mathrm{e}^{-\gamma \tau} U\left(z_{2}\right)-\beta U^{2}\left(z_{2}\right) .
\end{aligned}
$$

This means that $0 \leqslant U\left(z_{2}\right) \leqslant(\alpha / \beta) \exp (-\gamma \tau)$, which is a contradiction.

We now prove (iii), i.e. that the solution is monotone non-decreasing. Suppose this is false. Then, in view of the boundary conditions, the solution must have at least one local maximum. Let $z_{3}$ denote the leftmost such point, which is well defined since we know the decay to zero as $z \rightarrow-\infty$ is monotone. Now, since the solution must approach $(\alpha / \beta) \exp (-\gamma \tau)$ as $z \rightarrow \infty$, yet is confined to the range $[0,(\alpha / \beta) \exp (-\gamma \tau)]$, there must be a first point $z_{5}>z_{3}$ with $U^{\prime}\left(z_{5}\right)=0$ and $U^{\prime \prime}\left(z_{5}\right) \geqslant 0$. The reader should note that $z_{5}$ need not be a local minimum; it could, in principle, be a horizontal point of inflection (see figure 3, which illustrates some of the possibilities). There will also exist a point $z_{4}$ between $z_{3}$ and $z_{5}$ such that $U^{\prime}\left(z_{4}\right)<0$ and $U^{\prime \prime}\left(z_{4}\right)=0$, as shown in figure 3 . 


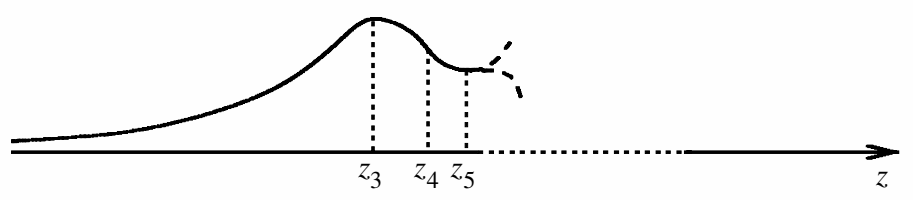

Figure 3. Graph illustrating how the solution $U(z)$ of (2.14) would lose monotonicity. The dashed parts suggest how it could behave for $z>z_{5}$. The conclusion is that, in fact, $U(z)$ must be monotone.

Note also that, by differentiation,

$$
U^{\prime \prime}(z)=\alpha \mathrm{e}^{-\gamma \tau} U^{\prime}(z-\tau)-2 \beta U(z) U^{\prime}(z) .
$$

From (2.15), when $z=z_{5}$, we see that $U^{\prime}\left(z_{5}-\tau\right) \geqslant 0$. This tells us $z_{5}-\tau \leqslant z_{3}$. Also, $U^{\prime \prime}\left(z_{4}\right)=0$ and $U^{\prime}\left(z_{4}\right)<0$, so, from $(2.15), U^{\prime}\left(z_{4}-\tau\right)<0$. But $z_{4}-\tau<z_{5}-\tau \leqslant z_{3}$, so we should have $U^{\prime}\left(z_{4}-\tau\right) \geqslant 0$, giving a contradiction. This completes the proof of the theorem.

\section{Global convergence}

In this section we shall formulate a reaction-diffusion extension of system (1.3) for the case of a finite spatial domain with homogeneous Neumann boundary conditions. Such boundary conditions model a closed environment with reflecting boundaries, i.e. individuals cannot leave the domain. It should be obvious that the delayed term $\alpha \mathrm{e}^{-\gamma \tau} u_{\mathrm{m}}(t-\tau)$ in system (1.3) cannot now be replaced by expression (1.5) because the arguments that led to that expression assumed the domain to be infinite. Where the argument breaks down is the assertion that the number born at $y$ that are still alive and now at $x$ will be given by multiplying the number born at $y$ and still alive by

$$
g(x, y, \tau)=\frac{1}{\sqrt{4 \pi d_{\mathrm{i}} \tau}} \mathrm{e}^{-(x-y)^{2} / 4 d_{\mathrm{i}} \tau} .
$$

As we explained earlier, expression (3.1) is the solution at time $t=\tau$ of $\partial g / \partial t=$ $d_{\mathrm{i}} \partial^{2} g / \partial x^{2},-\infty<x<\infty$, with $g(x, y, 0)=\delta(x-y)$. For the case of a finite domain, which can be taken without loss of generality to be $0 \leqslant x \leqslant \pi$, the modification that needs to be made is to replace (3.1) by $G(x, y, \tau)$, where $G(x, y, t)$ is the solution of

$$
\frac{\partial G}{\partial t}=d_{\mathrm{i}} \frac{\partial^{2} G}{\partial x^{2}}, \quad 0<x<\pi
$$

subject to

$$
\frac{\partial G}{\partial x}=0 \quad \text { at } x=0, \pi \quad \text { and } \quad G(x, y, 0)=\delta(x-y) .
$$

Solving (3.2), (3.3) gives

$$
G(x, y, t)=\frac{1}{\pi}+\frac{2}{\pi} \sum_{n=1}^{\infty} \mathrm{e}^{-d_{\mathrm{i}} n^{2} t} \cos n x \cos n y .
$$


Note that the function $G(x, y, t)>0$ for all $x, y$ if $t>0$. The reader should further note that $G$, defined by (3.4), also satisfies (3.2), (3.3), with $x$ and $y$ interchanged.

Thus, for the case of a finite domain $0 \leqslant x \leqslant \pi$, the second equation of (1.6) will be replaced by

$$
\frac{\partial u_{\mathrm{m}}}{\partial t}=d_{\mathrm{m}} \frac{\partial^{2} u_{\mathrm{m}}}{\partial x^{2}}+\alpha \mathrm{e}^{-\gamma \tau} \bar{u}_{\mathrm{m}}(x, t)-\beta u_{\mathrm{m}}^{2}, \quad t>0, \quad 0<x<\pi,
$$

where

$$
\bar{u}_{\mathrm{m}}(x, t)=\int_{0}^{\pi} G(x, y, \tau) u_{\mathrm{m}}(y, t-\tau) \mathrm{d} y,
$$

with $G$ given by (3.4), and with boundary conditions

$$
\frac{\partial u_{\mathrm{m}}}{\partial x}(0, t)=\frac{\partial u_{\mathrm{m}}}{\partial x}(\pi, t)=0, \quad t>0,
$$

and initial condition

$$
u_{\mathrm{m}}(x, t)=\phi(x, t) \quad \text { for }(x, t) \in[0, \pi] \times[-\tau, 0] .
$$

Note that if $u_{\mathrm{m}}(x, s) \geqslant 0$ for all $x \in[0, \pi]$ and $s \leqslant t$, then $\bar{u}_{\mathrm{m}}(x, t) \geqslant 0$. This follows from the positivity of $G$. Note also that $G$ satisfies

$$
\int_{0}^{\pi} G(x, y, t) \mathrm{d} y=1
$$

for any $t \geqslant 0$ and, in particular, $t=\tau$. Thus $\bar{u}_{\mathrm{m}}$ is a weighted average of $u_{\mathrm{m}}$ at an earlier time.

We prove that all ecologically relevant solutions $u_{\mathrm{m}}(x, t)$ approach $(\alpha / \beta) \exp (-\gamma \tau)$ as $t \rightarrow \infty$, uniformly for $x \in[0, \pi]$. This holds without any restriction on the parameters other than that they be positive. The key to proving our theorem is that a comparison theorem holds for the scalar equation (3.5). This can be seen from the theory developed by Redlinger (1984) for parabolic equations and systems containing functional terms, and also from the abstract approach developed in Martin \& Smith (1990) (see, in particular, proposition 3, remark 2.4 and corollary 5 of that paper). In the Redlinger (1984) approach, a pair of sub/supersolutions for the problem (3.5)-(3.8) is a pair of functions $v$ and $w$, which are continuous on $[0, \pi] \times[-\tau, \infty)$, possess all partial derivatives referred to in (ii) below (the existence of $v_{t}$ and $w_{t}$ as one-sided derivatives from below being sufficient) and

(i) $v \leqslant w$ for $(x, t) \in[0, \pi] \times[-\tau, \infty)$;

(ii) $v_{t} \leqslant d_{\mathrm{m}} v_{x x}+\alpha \mathrm{e}^{-\gamma \tau} \bar{\psi}-\beta v^{2}$ and $w_{t} \geqslant d_{\mathrm{m}} w_{x x}+\alpha \mathrm{e}^{-\gamma \tau} \bar{\psi}-\beta w^{2}$ in $(0, \pi) \times(0, \infty)$, for all continuous functions $\psi$ such that $v \leqslant \psi \leqslant w$ in $[0, \pi] \times[-\tau, \infty)$;

(iii) $v_{x}(0, t) \geqslant 0, v_{x}(\pi, t) \leqslant 0, w_{x}(0, t) \leqslant 0$ and $w_{x}(\pi, t) \geqslant 0$;

(iv) $v(x, s) \leqslant \phi(x, s) \leqslant w(x, s)$ for $(x, s) \in[0, \pi] \times[-\tau, 0]$.

The following lemma then follows from theorem 3.4 in Redlinger (1984).

Lemma 3.1. Let $v$ and $w$ be a pair of sub/supersolutions for (3.5)-(3.8) and suppose that the initial function $\phi$ is Hölder continuous in $[0, \pi] \times[-\tau, 0]$. Then (3.5)-(3.8) has exactly one regular solution $u_{\mathrm{m}}(x, t)$ satisfying $v \leqslant u_{\mathrm{m}} \leqslant w$ in $[0, \pi] \times[-\tau, \infty)$. 
It is, of course, enough to verify the two inequalities in (ii) above for $\psi=v$ and $\psi=w$, respectively. This is because, in (3.5), the reaction term is increasing with respect to the delayed variable $\bar{u}_{\mathrm{m}}(x, t)$. The same observation would, of course, apply to any other reaction term having this property.

We may now state the following result.

Theorem 3.2. Let the initial function $\phi$ be Hölder continuous in $[0, \pi] \times[-\tau, 0]$ and satisfy $\phi \geqslant 0$ with $\phi \not \equiv 0$. Assume that $\alpha, \beta>0$ and $\gamma, \tau \geqslant 0$. Then problem (3.5)(3.8) has a unique positive solution $u_{\mathrm{m}}(x, t)$ satisfying

$$
u_{\mathrm{m}}(x, t) \rightarrow \frac{\alpha}{\beta} \mathrm{e}^{-\gamma \tau} \quad \text { as } t \rightarrow \infty
$$

uniformly for $x \in[0, \pi]$.

Proof. By the foregoing remarks, any solution of the second equation of (1.3) can be a sub/supersolution of (3.5)-(3.8). First note that if $\tau=0$, then $\bar{u}_{\mathrm{m}}(x, t)=$ $u_{\mathrm{m}}(x, t)$ (since $G(x, y, 0)=\delta(x-y)$ ) and the problem reduces to Fisher's equation for which the conclusion of the theorem is already well known. Therefore, we assume that $\tau>0$.

It is readily seen that $(0, K)$ are a sub/supersolution pair, where

$$
K=\max \left(\frac{\alpha}{\beta} \mathrm{e}^{-\gamma \tau}, \max \{\phi(x, s), x \in[0, \pi], s \in[-\tau, 0]\}\right) .
$$

Hence $0 \leqslant u_{\mathrm{m}}(x, t) \leqslant K$. We now aim to show that $u_{\mathrm{m}}(x, t)>0$ for $t$ sufficiently large (in particular, for all $t>\tau$ ). Recall that $\phi$ satisfies $\phi \geqslant 0$ with $\phi \not \equiv 0$. There are two cases to consider. The first is when $\phi(x, 0) \not \equiv 0$ and we claim that in this case $u_{\mathrm{m}}(x, t)>0$ on $[0, \pi] \times(0, \infty)$. Suppose, on the contrary, that there exist $\left(x^{*}, t^{*}\right)$ in $[0, \pi] \times(0, \infty)$ with $u_{\mathrm{m}}\left(x^{*}, t^{*}\right)=0$. Then, since $0 \leqslant u_{\mathrm{m}} \leqslant K$, it is readily seen that, for $0<t \leqslant t^{*}, u_{\mathrm{m}}$ can be interpreted as satisfying a linear parabolic inequality

$$
\frac{\partial u_{\mathrm{m}}}{\partial t} \geqslant d_{\mathrm{m}} \frac{\partial^{2} u_{\mathrm{m}}}{\partial x^{2}}+h(x, t) u_{\mathrm{m}}
$$

with $h$ being a bounded function. Under these circumstances, the strong-maximum principle and boundary-point lemma as described in Britton (1986) immediately yield that $\phi(x, 0) \equiv 0$, which is a contradiction. The second case to consider is when $\phi(x, 0) \equiv 0$. We claim that the solution of $(3.5)-(3.8)$ cannot be identically zero on $[0, \pi] \times(0, \tau]$. Indeed, if this were false, then, since the governing equation on $0<t \leqslant \tau$ is

$$
\frac{\partial u_{\mathrm{m}}}{\partial t}=d_{\mathrm{m}} \frac{\partial^{2} u_{\mathrm{m}}}{\partial x^{2}}+\alpha \mathrm{e}^{-\gamma \tau} \int_{0}^{\pi} G(x, y, \tau) \phi(y, t-\tau) \mathrm{d} y-\beta u_{\mathrm{m}}^{2}, \quad 0<x<\pi,
$$

we would conclude that

$$
\int_{0}^{\pi} G(x, y, \tau) \phi(y, t-\tau) \mathrm{d} y=0, \quad \text { for all }(x, t) \in[0, \pi] \times(0, \tau],
$$

which contradicts $\phi \not \equiv 0$ (recall that $G(x, y, \tau)$ is strictly positive). So there exists $t^{* *} \in(0, \tau]$ with $u_{\mathrm{m}}\left(\cdot, t^{* *}\right) \not \equiv 0$. Using similar arguments to the previous case, we can show that $u_{\mathrm{m}}(x, t)>0$ for all $(x, t) \in[0, \pi] \times\left(t^{* *}, \infty\right)$. 
Choose any $T$ such that $T>\tau$. Then $\min \left\{u_{\mathrm{m}}(x, t), x \in[0, \pi], t \in[T, T+\tau]\right\}>0$. The initial time (i.e. the time $t=0$ in the foregoing remarks) shall henceforth be taken as $T+\tau$, with conditions (i)-(iv) above amended appropriately. Let $v(t)$ and $w(t)$ be the continuous functions defined by

$$
\left.\begin{array}{rlrl}
v(t) & =\min \left\{u_{\mathrm{m}}(x, s), x \in[0, \pi], s \in[T, T+\tau]\right\}, & & T \leqslant t \leqslant T+\tau, \\
\dot{v}(t) & =\alpha \mathrm{e}^{-\gamma \tau} v(t-\tau)-\beta v^{2}(t), & & t>T+\tau,
\end{array}\right\}
$$

and

$$
\left.\begin{array}{ll}
w(t):=\max \left\{u_{\mathrm{m}}(x, s), x \in[0, \pi], s \in[T, T+\tau]\right\}, & T \leqslant t \leqslant T+\tau, \\
\dot{w}(t)=\alpha \mathrm{e}^{-\gamma \tau} w(t-\tau)-\beta w^{2}(t), & t>T+\tau .
\end{array}\right\}
$$

Then $v$ and $w$ are a sub/supersolution pair and thus $v(t) \leqslant u_{\mathrm{m}}(x, t) \leqslant w(t)$ in $[0, \pi] \times[T, \infty)$. But all solutions of the second equation of (1.3) approach $(\alpha / \beta) \exp (-\gamma \tau)$ as $t \rightarrow \infty$ (see Aiello \& Freedman 1990). Hence (3.9) holds. The proof of the theorem is complete.

Finally, we shall discuss the convergence of the immature population $u_{\mathrm{i}}$. For the finite domain $x \in[0, \pi]$, the first equation of (1.6) will become

$$
\frac{\partial u_{\mathrm{i}}}{\partial t}=d_{\mathrm{i}} \frac{\partial^{2} u_{\mathrm{i}}}{\partial x^{2}}+\alpha u_{\mathrm{m}}-\gamma u_{\mathrm{i}}-\alpha \mathrm{e}^{-\gamma \tau} \bar{u}_{\mathrm{m}}(x, t)
$$

with

$$
\bar{u}_{\mathrm{m}}(x, t)=\int_{0}^{\pi} G(x, y, \tau) u_{\mathrm{m}}(y, t-\tau) \mathrm{d} y
$$

and $G$ again defined by (3.4). Since $u_{\mathrm{m}}(t)$ can now be regarded as a known function, the comparison theorem for equations without delay (see, for example, Smith 1995, theorem 3.4) can be applied to (3.12) under homogeneous Neumann boundary conditions.

Let $\varepsilon>0$ be arbitrary. By the uniform convergence of $u_{\mathrm{m}}(x, t)$ proved in theorem 3.2 , it is true that, for all $t$ sufficiently large,

$$
u_{\mathrm{m}}(x, t), \bar{u}_{\mathrm{m}}(x, t) \in\left[\frac{\alpha}{\beta} \mathrm{e}^{-\gamma \tau}-\varepsilon, \frac{\alpha}{\beta} \mathrm{e}^{-\gamma \tau}+\varepsilon\right] .
$$

Without going into the technicalities concerning initial conditions, it follows that, if the initial time is suitably large, a solution $u_{\mathrm{i}}^{+}(t)$ of

$$
\frac{\mathrm{d} u_{\mathrm{i}}^{+}}{\mathrm{d} t}=\frac{\alpha^{2}}{\beta} \mathrm{e}^{-\gamma \tau}\left(1-\mathrm{e}^{-\gamma \tau}\right)+\varepsilon \alpha\left(1+\mathrm{e}^{-\gamma \tau}\right)-\gamma u_{\mathrm{i}}^{+},
$$

with appropriate 'initial condition', is a supersolution for (3.12). Also, a suitable solution $u_{\mathrm{i}}^{-}(t)$ of

$$
\frac{\mathrm{d} u_{\mathrm{i}}^{-}}{\mathrm{d} t}=\frac{\alpha^{2}}{\beta} \mathrm{e}^{-\gamma \tau}\left(1-\mathrm{e}^{-\gamma \tau}\right)-\varepsilon \alpha\left(1+\mathrm{e}^{-\gamma \tau}\right)-\gamma u_{\mathrm{i}}^{-}
$$


is a subsolution. Hence, for sufficiently large $t$,

$$
u_{\mathrm{i}}^{-}(t) \leqslant u_{\mathrm{i}}(x, t) \leqslant u_{\mathrm{i}}^{+}(t) .
$$

Hence

$$
\begin{aligned}
\liminf _{t \rightarrow \infty} \min \left\{u_{\mathrm{i}}(x, t), x \in[0, \pi]\right\} & \geqslant \lim _{t \rightarrow \infty} u_{\mathrm{i}}^{-}(t) \\
& =\frac{1}{\gamma}\left(\frac{\alpha^{2}}{\beta} \mathrm{e}^{-\gamma \tau}\left(1-\mathrm{e}^{-\gamma \tau}\right)-\varepsilon \alpha\left(1+\mathrm{e}^{-\gamma \tau}\right)\right) .
\end{aligned}
$$

Similarly,

$$
\begin{aligned}
\limsup _{t \rightarrow \infty} \max \left\{u_{\mathrm{i}}(x, t), x \in[0, \pi]\right\} & \leqslant \lim _{t \rightarrow \infty} u_{\mathrm{i}}^{+}(t) \\
& =\frac{1}{\gamma}\left(\frac{\alpha^{2}}{\beta} \mathrm{e}^{-\gamma \tau}\left(1-\mathrm{e}^{-\gamma \tau}\right)+\varepsilon \alpha\left(1+\mathrm{e}^{-\gamma \tau}\right)\right) .
\end{aligned}
$$

Since these hold for all sufficiently small $\varepsilon>0$, we conclude that

$$
u_{\mathrm{i}}(x, t) \rightarrow \frac{\alpha^{2}}{\beta \gamma} \mathrm{e}^{-\gamma \tau}\left(1-\mathrm{e}^{-\gamma \tau}\right) \quad \text { as } t \rightarrow \infty,
$$

uniformly for $x \in[0, \pi]$.

\section{Discussion}

There are quite a few mathematical treatments of the diffusive delay logistic equation and Nicholson's blowflies equation (Gourley 2000a,b; So et al. 2001). The diffusive process of the population seems to enrich the dynamics for these two delay models. These models assume a constant death rate and, as a result, wavefronts can be shown to exist and their properties can be obtained. Through the use of a sub/supersolution pair method, we have shown that the diffusive delay version of model (1.3) continues to produce simple global dynamics. We have also established the monotone property of any possible wavefront solution of (2.12). However, the existence of wavefront solutions for this model remains an open question.

Without diffusion, the delay differential model in question is a direct extension of the well-known logistic differential equation with delayed constant birth processes and instantaneous quadratically regulated death processes. In general, a singlespecies population growth with two-stage structure can be modelled as follows,

$$
y^{\prime}(t)=\mathrm{e}^{-d \tau} B(y(t-\tau))-D(y(t)),
$$

where $B(y)$ is the birth rate, $d$ is the juvenile through-stage death rate, $D(y)$ is the death term and $\tau$ is the time taken from birth to maturity. We say that the growth process is regulated by strong death process if $\lim _{y \rightarrow+\infty} D(y) / y=+\infty$. In particular, if $\lim _{y \rightarrow+\infty} D(y) / y^{2}=c$ for some positive constant $c$, then we say it is a quadratically regulated death process. Model (1.3) assumes constant birth rate $(B(y)=\alpha y)$ and quadratic death process. It is known (Kuang 1993, theorem 4.9.1) that if both functions $B(y)$ and $D(y)$ are strictly increasing and the model (4.1) admits a unique positive steady state, then this delay-dependent steady state is a global attractor, regardless of the delay length. We believe that when diffusion is properly introduced 
and the reaction term adjusted accordingly, a diffusive version of model (4.1) may continue to produce such simple global dynamics. If growth is regulated by strong instantaneous death process, then the existence of wavefronts, though intuitively very likely, may be non-trivial to establish. In contrast, if growth is regulated by the birth process $\left(\lim _{y \rightarrow+\infty} B(y) / y=0\right)$ and the death rate is constant, as in the Nicholson's blowflies model, then the dynamics is more colourful and qualitatively dependent on the time delay (positive steady state, if it exists, may be stable for both small and large delay, and unstable for intermediate delay (see Beretta \& Kuang 2002; Cooke et al. 1999)), and the wavefront solution can be established by the method of So et al. (2001).

The above comments have the following important biological implications.

(i) Strongly regulated death process dramatically simplifies the growth dynamics.

(ii) Birth-regulated growth processes can generate rich dynamics due to the maturation delay.

(iii) When modelling a single-species population growth, the birth and death processes are two key factors, their importance exceeding that of diffusive movement and the effect of maturation time delay.

We are grateful to the referees for their suggestions, which helped to improve this paper. The work of Y.K. is partly supported by NSF grant DMS-0077790.

\section{References}

Aiello, W. G. \& Freedman, H. I. 1990 A time-delay model of single species growth with stage structure. Math. Biosci. 101, 139-153.

Aiello, W. G., Freedman, H. I. \& Wu, J. 1992 A model of stage structured population growth with density dependent time delay. SIAM J. Appl. Math. 52, 855-869.

Ashwin, P., Bartuccelli, M. V., Bridges, T. J. \& Gourley, S. A. 2002 Travelling fronts for the KPP equation with spatio-temporal delay. Z. Angew. Math. Phys. 53, 103-122.

Beretta, E. \& Kuang, Y. 2002 Geometric stability switch criteria in delay differential systems with delay dependent parameters. SIAM J. Math. Analysis 33, 1144-1165.

Britton, N. F. 1986 Reaction-diffusion equations and their applications to biology. Academic.

Canosa, J. 1973 On a nonlinear diffusion equation describing population growth. IBM J. Res. Develop. 17, 307-313.

Cooke, K. L., van den Driessche, P. \& Zou, X. 1999 Interaction of maturation delay and nonlinear birth in population and epidemic models. J. Math. Biol. 39, 332-352.

Gourley, S. A. $2000 a$ Travelling front solutions of a nonlocal Fisher equation. J. Math. Biol. 41, $272-284$.

Gourley, S. A. $2000 b$ Travelling fronts in the diffusive Nicholson's blowflies equation with distributed delays. Math. Comput. Modelling 32, 843-853.

Gourley, S. A. \& Britton, N. F. 1996 A predator prey reaction diffusion system with nonlocal effects. J. Math. Biol. 34, 297-333.

Kuang, Y. 1993 Delay differential equations with applications in population dynamics. Academic.

Martin, R. H. \& Smith, H. 1990 Abstract functional differential equations and reaction-diffusion systems. Trans. Am. Math. Soc. 321, 1-44.

Murray, J. D. 1989 Mathematical biology. Springer.

Redlinger, R. 1984 Existence theorems for semilinear parabolic systems with functionals. Nonlin. Analysis 8, 667-682.

Proc. R. Soc. Lond. A (2003) 
Sherratt, J. A. 2000 Wavefront propagation in a competition equation with a new motility term modelling contact inhibition between cell populations. Proc. R. Soc. Lond. A 456, 2365-2386.

Smith, H. L. 1995 Monotone dynamical systems: an introduction to the theory of competitive and cooperative systems. Providence, RI: American Mathematical Society.

So, J. W.-H., Wu, J. \& Zou, X. 2001 A reaction diffusion model for a single species with age structure I. Travelling wave fronts on unbounded domains. Proc. R. Soc. Lond. A 457, 18411853. 